\title{
Design of Distributed Electricity Trading Mechanism Based on Contract for Difference
}

\author{
Li Long ${ }^{1}$, Tianhai Yang ${ }^{2}$, Qifen $\mathrm{Li}^{*}$, Yongwen Yang ${ }^{3}$, Lifei Song ${ }^{3}$ and Tianjiao $\mathrm{Wu}^{3}$ \\ ${ }^{1}$ Shanghai University of Electricity Power, Shanghai, 200090, China; \\ ${ }^{2}$ Shanghai Minghua Electric Power Technology Co., Ltd., Shanghai, 200090, China; \\ *Shanghai Power Equipment Research Institute Co., Ltd., Shanghai, 200090 \\ ${ }^{3}$ Shanghai University of Electricity Power, Shanghai, 200090, China
}

\begin{abstract}
A contract for difference is a medium and long-term financial contract, which can be used in the electricity market to lock the electricity price in advance and avoid the risk of electricity price fluctuations in the spot market. The construction of the domestic power spot market has just started. With the release of relevant policies and the gradual improvement of the market structure, it is urgent to design a corresponding trading mechanism to ensure the smooth transition of the market. The current day-ahead transactions, realtime transactions and other short-term transactions for distributed power generation, on the one hand power load forecasting, electricity price demand response and other related technologies need to be further improved, on the other hand due to the randomness and uncertainty of distributed energy, participating in the short-term spot market has large price fluctuations, which is not conducive to the stability of the electricity market, and it is also not conducive to the consumption of distributed energy. Aiming at the above problems, this paper uses the characteristics of CFDs to restrain market power to design a distributed energy trading mechanism to achieve the purpose of energy saving and emission reduction.
\end{abstract}

\section{Introduction}

With the deepening of a new round of power reform in China, new requirements have been put forward for the power trading market. In accordance with the requirements of the Opinions on Further Deepening the Reform of the Power System (Zhongfa No. 9 of 2015) and related supporting documents, with the goal of restoring the commodity properties of electric power, the market mechanism optimizes the allocation of power resources, and gradually establishes a market-oriented power balance mechanism with medium- and long-term trading as the main focus and spot trading as the supplement ${ }^{[1]}$.At present, China's electricity market model is still in the state investment and government control of power generation, transmission, distribution, electricity monopoly integration model, along with the introduction of power side reform, China's electricity market is currently changing to the retail competition mode ${ }^{[4]}$. In recent years, scholars at home and abroad have carried out a lot of research work in the marketization of distributed power generation, and have made some research results. Literature ${ }^{[5-9]}$ Considering the price mechanism of the electricity market, the price model of power transaction is established to reduce the user's power generation cost, increase the benefit of the power generation side cost unit, and promote the fair competition of the market. Literature ${ }^{[10-12]}$. This paper summarizes and analyzes the domestic and foreign power spot market, and provides the theoretical basis for the development of China's electric power spot market by studying the foreign power spot market. At the same time, from the power financial derivatives transactions, by drawing on the financial market trading model, to promote fair competition in the electricity market, Literature ${ }^{[13-14]}$. The application of foreign CFD trading in the power market is reviewed and analyzed, which provides a theoretical basis for the design of CFD mechanism in the construction of China's power market. Literature ${ }^{[15]}$. From the point of view of power futures trading, the forward contracts and bilateral contracts of the power market are briefly summarized, and the characteristics of strong market stability of futures trading are also worthy of reference in the spot market. From the point of view of power futures trading, the forward contracts and bilateral contracts of the power market are briefly summarized, and the characteristics of strong market stability of futures trading are also worthy of reference in the spot market.

\section{Analysis of the status of distributed transactions}

\subsection{The need for distributed energy trading}

At present, the concept of distributed trading, the relevant literature at home and abroad to its definition is more

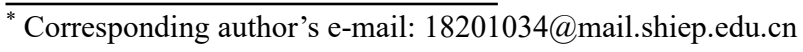


consistent, that is, to break the original power system structure, distributed power generation to carry out market-oriented transactions, to achieve distributed power in a certain area, a certain voltage level of neardisappearing.

According to the requirements of the Notice and the relevant literature research, distributed transactions mainly have the following characteristics:

(1) The limit of the transmission voltage.

The Notice stipulates that $110 \mathrm{KV}$ is the high point of distributed project market trading, which means that the scope of construction of distributed power stations must not be greater than $100 \mathrm{~km}$.

(2) Direct transaction between supply and demand: when distributed power generation has not yet been involved in the power market, distributed power generation will transmit the full amount of power generated by its own units to the end of the large power grid, by the large grid for unified scheduling, consumers do not have the right to choose electricity, and distributed transactions give both sides of supply and demand a certain choice, the seller can choose the high price of consumers or can fully eliminate their electricity supply consumers to trade, consumers can also choose their own electricity supply according to their own electricity demand.

(3) More conducive to stimulate the competitiveness of the distributed energy market, after participating in distributed transactions, DG can obtain legitimate flashlight qualification, with the initiative to establish the "sale and distribution of storage" integration and participation in the grid auxiliary services market power, with the traditional power companies or distributed power generation, will have an absolute competitive advantage.

\subsection{The current problem of distributed transactions}

(1) Distributed energy installed capacity has brought a series of problems. On the one hand, the energy storage pressure is greater, on the other hand, the phenomenon of wind abandonment is serious, and the problem of distributed energy consumption is becoming more and more prominent.

(2) The quality of the distributed power generation companies is uneven, and it is necessary to create a good market environment for the distributed power generation market through the survival of the market competition.
(3) Distributed power generation due to its own characteristics lead to power generation randomness, instability, direct participation in power market transactions is not competitive, and the relevant distributed technology is not complete before the stability of the power market.

\section{Trade flow design based on CFDs}

\subsection{Analysis of the suitability of distributed energy trading and CFDs}

The power medium- and long-term trading market forms two forms of contract: physical contracts and CFDs. The physical contract signed in the medium- and long-term market needs to be actually executed, and how much electricity will be used to sign the contract. CFDs signed in medium- and long-term trading do not require physical execution, but are settled only by the difference between spot market electricity prices and contract prices. CFDs are a financial tool that affects only settlement. The electricity market introduces CFDs. There are two effects:

1. Because CFDs do not require physical execution in the spot market, it will not constrain the optimal allocation of resources in the spot market. For example: A generator set quotation is lower than $\mathrm{B}$ generator set, $\mathrm{B}$ generator set can choose not to generate electricity, low quotation unit A power generation. Unit B buys low-cost spot electricity from Unit A and completes its own medium- and longterm contracts through the CFD settlement mechanism. The same is true for the user side, users can buy low and sell high spot electricity, in the performance of mediumand long-term contracts under the premise of protecting or even expanding their own interests.

2. In the quotation, the user side wants the lower the electricity price is the better, the power generation side wants the higher the electricity price the better. CFDs can effectively avoid low or high spot market quotes. The parties have discussed in advance and privately the volume of the contract (which may not pass through the trading center) and the contract price, and signed the contract. At the time of settlement, through the ratio of medium- and long-term contracts to the spot market, the contract is settled according to the contract price, the contract is settled at the spot market price outside the contract, the contract is not enough electricity can be bought in the spot market, the contract is signed with excess electricity can be sold in the spot market.

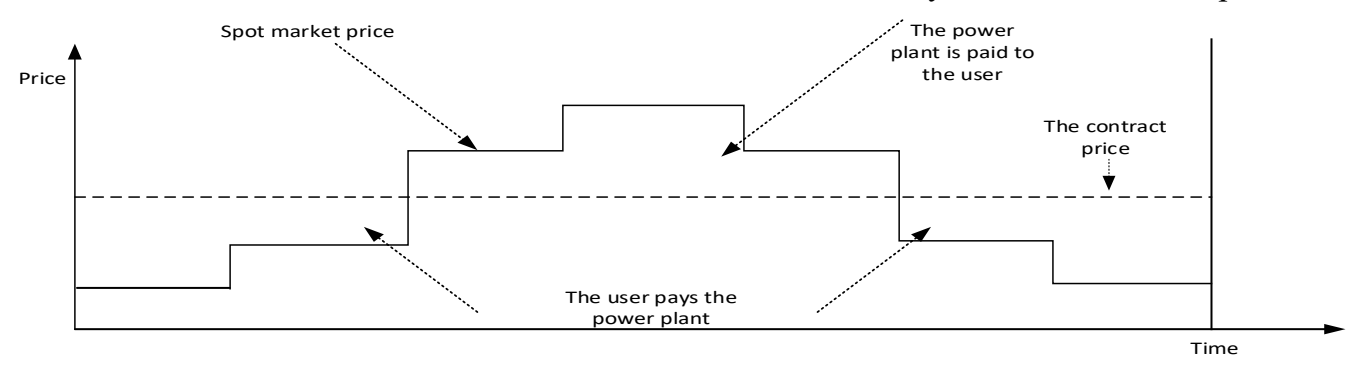

Figure 1: A diagram of CFDs

If the spot market price is higher than the CFD price, the power generation side will be refunded to the user side 
of the corresponding electricity charges, if the spot market price is lower than the CFD price, the user side will pay the corresponding power charges on the power generation side. Therefore, the judgment of CFDs and spot market prices is directly related to the actual interests.

For both parties, both are conducive to the stability of electricity prices, is a good mechanism to avoid price risk.

\subsection{The design of the transaction process}

Because of the uncertainty and difference of distributed power generation, as well as the random fluctuation of real-time electricity price, distributed energy has no advantage in the power trading market, the traditional power trading mechanism can not meet the requirements of distributed power generation, so it is necessary to design a power market trading mechanism to support distributed energy consumption and improve the power market transaction.

The distributed power trading market participants in this paper include energy sellers, energy users and energy trading centers. Trading is through online bilateral negotiations and contract transactions. The entire trading process is shown in Figure 1.

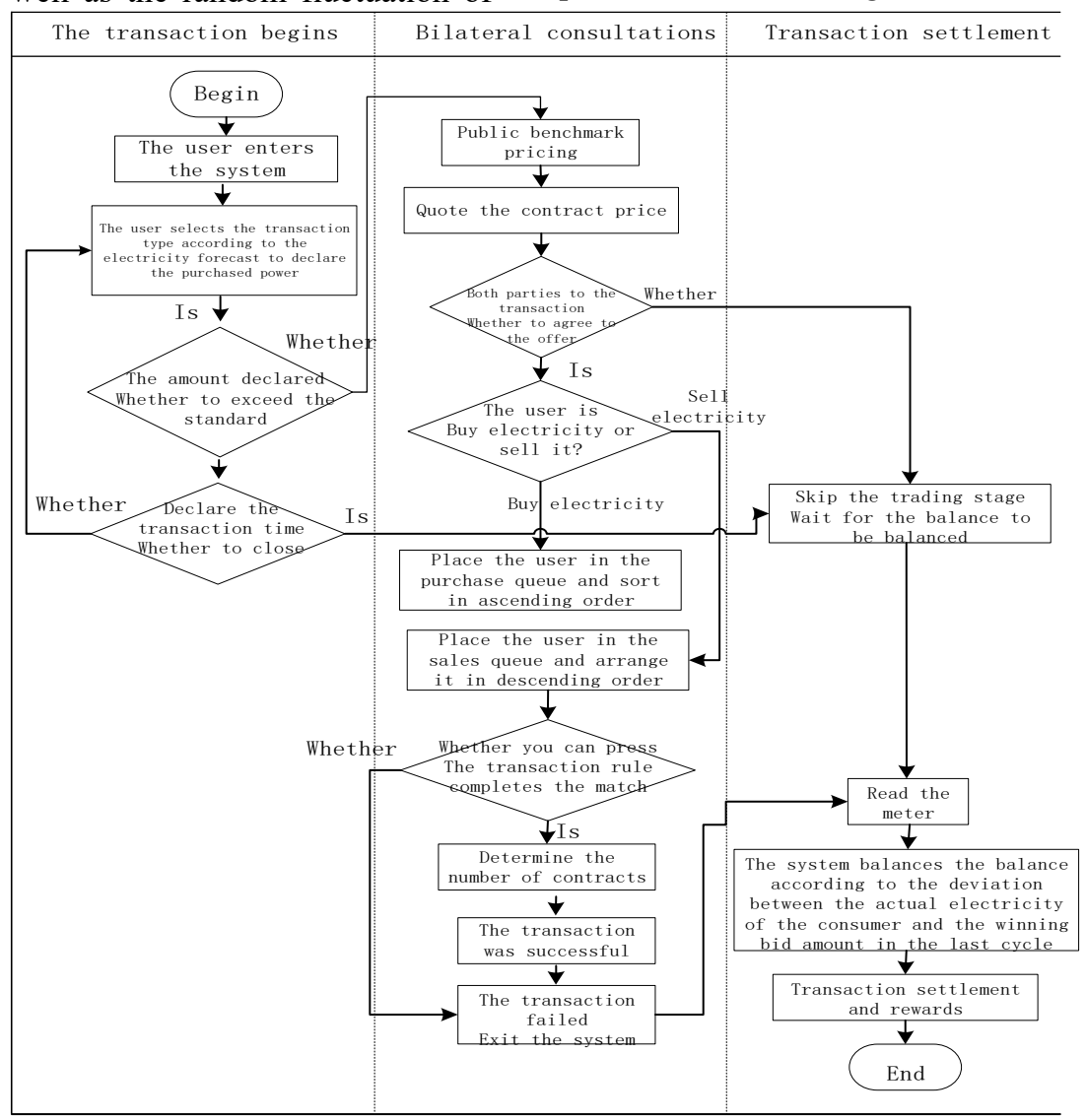

Fig.2 Transaction flowchart of distribution network

\subsection{The implementation of the transaction process}

1) Before the start of the transaction, the user first of all their own electricity demand forecast assessment, into the system, according to the planned electricity consumption to choose the type of energy traded, each energy will have a different subsidy policy, users according to their own circumstances to choose a different power generator

2) If the user can accept the price provided by the energy supplier, then enter the queuing sequence in chronological order, the energy power generator participating in the transaction is sorted according to the order in which the transaction was registered, and the transaction order is in the order of queuing, ranking first in the front end of the transaction.

At the same time, the system will test the user's declaration quantity, if the user's declaration amount exceeds its constraints, in a valid time needs to re-report the demand. After the trading time, skip the trading stage and go directly to the balance balance stage. The user declares the battery constraint.

$$
p_{n} \leq p_{n, \max }, \forall n \in \Omega_{p}
$$

The above calculation: $P_{n, \max }$ is the upper limit of the load of the nth generator; $\Omega_{p}$ is A collection for power generators.

3) Under the premise of meeting the requirements of rationality, fairness, economy and risk, the annual contract power is broken down into each generator set in the spatial dimension, and the initial decomposition scheme of the contract power is obtained by breaking down from the annual to the monthly, monthly to the daily and finally into the time period. The initial decomposition process is as 
follows:

Step 1: Analysis and clustering of historical load curves, and comprehensive consideration of meteorological, holiday, yin and yang calendar and other major factors, after the revision of the typical load curve;

Step 2: Forecast annual electricity consumption, and in accordance with the prescribed market opening ratio, taking into account factors such as plant electricity consumption, power grid loss and other factors, to find annual CFD electricity;

Step 3: To determine the annual contract power of each generator set by taking the power generation capacity of the unit as the basis for the decomposition of CFD power in the spatial dimension;

Step 4: According to the typical load curve, CFD power is decomposed in the time dimension, that is, from the annual breakdown to the monthly, daily, and time periods.

Step 5: Rolling correction is carried out on the basis of the preliminary decomposition results obtained by the proportional decomposition method, taking into account such factors as generator set maintenance, shutdown, cogeneration, power generation deviation, and safe and stable operation constraints of the power system.

4) The system reads the power transaction of the last cycle, balances the amount of the bid in the last cycle and the actual amount of electricity issued/used, the public power grid will balance the deviation of the electricity, and the deviation of the current cycle will balance the deviation of the electricity in the next cycle.

5) The system automatically settles and rewards this cycle trading according to the rules, and the trading platform settles the reward according to the amount declared and the actual electricity generated/used by the user participating in the transaction in the last cycle, and the reward for this cycle will be carried out in the next cycle.

\section{Conclusion}

(1) This paper analyzes the existing problems of distributed energy transactions, on the one hand, distributed energy installed capacity is large, the problem needs to be solved urgently, on the other hand, there are many distributed power generation companies, lack of market competition in the field of distributed energy, is not conducive to the development of distributed energy. At present, there is an urgent need for a reasonable trading mechanism, which can not only find the market price of distributed energy trading market, but also promote the consumption of distributed energy and promote the development of distributed energy.

(2) This paper introduces the CFD mechanism, combined with distributed energy trading analysis, CFDs have a dampening effect on the market, can prevent price fluctuations in the spot market. It has a great effect on promoting distributed energy trading, which is still in the exploration stage.

(3) This paper designs the CFD trading mechanism, combined with the characteristics of distributed energy trading, so that distributed energy trading can be better implemented. This trading mechanism make the power market run steadily.

\section{References}

1. Lin Wei, Xu Bingxuan, Wang Weihuai. Analysis and suggestion of the market-oriented trading mechanism of typical distributed power generation [J]. Power system automation, 2019, 43(04):1-8.

2. Shi Gangsong, Zhang Jing, Li Bowen, etc. Market equilibrium study of bidding for electricity under the coexistence of multi-type power sales companies $[\mathrm{J}]$. Power system protection and control, 2018, 46(5): 62-67.

3. Wang Haohao, Wang Yi, Zhang Wei, Duan Qingang, Koo Weihui, Chen Qixin. Analysis of game equilibrium in the power market for different bidding mechanisms [J]. Power demand side management,2020,22(04):2-7.

4. Ding Xinhai. Research on the settlement price mechanism of bidding transactions in the power market [J]. Hua zhong electric power,2005(01):21$24+36$.

5. Wang Zuoquan, Cao Xuexuan. To improve the price mechanism of electric power market transaction and its supervision $[\mathrm{J}]$. Price theory and practice,2018(04):26-29.

6. Fan Yuhong, Ding Wei, Zhang Wei, Xu Qiushi. Research on the key issues of power spot market construction [J]. Hubei Electric Power, 2018, 42(02): 45-50.

7. Zhang Yi, Song Xinming, Liu Weiguo, Liu Wei. A comparative analysis of the two price mechanisms in the power market to inhibit the exercise of market forces [J]. Grid technology,2006(S2):619623.

8. Wang Mingyuan. A comparative study of the typical electric power spot market abroad [J]. Integrated circuit applications,2020,37(08):98-99.

9. Cui Yayi. Reflections on new energy access under the market-oriented reform of power trading [J].2020, 41(03):387-388 